\title{
Cytogenetics of the Brazilian Bolitoglossa paraensis (Unterstein, 1930) salamanders (Caudata, Plethodontidae)
}

\author{
Jéssica Barata da Silva ${ }^{1}$, Pablo Suárez ${ }^{1}$, Cleusa Yoshiko Nagamachi ${ }^{1}$, Timothy Frederick Carter ${ }^{2}$ \\ and Julio Cesar Pieczarka ${ }^{1}$ \\ ${ }^{1}$ Laboratorio de Citogenética, Instituto de Ciências Biológicas, Universidade Federal do Pará, \\ Belém, PA, Brazil. \\ ${ }^{2}$ Department of Biomedical Sciences, Ontario Veterinary College, University of Guelph, Guelph, \\ ON, Canada.
}

\begin{abstract}
Plethodontid salamanders of genus Bolitoglossa constitute the largest and most diverse group of salamanders, including around $20 \%$ of living caudate species. Recent studies have indicated the occurrence of five recognized species in the Brazilian Amazon Rainforest. We present here the first cytogenetic data of a Brazilian salamander, which may prove to be a useful by contribution to the cytotaxonomy of the genus. Specimens were collected near the "type" locality (Utinga, Belém, PA, Brazil). Chromosomal preparations from duodenal epithelial cells and testes were subjected to Giemsa staining, C-banding and DAPI/CMA fluorochrome staining. All specimens showed a karyotype with 13 bi-armed chromosome pairs $(2 n=26)$. Nucleolar Organizer Regions, evidenced by $\mathrm{CMA}_{3}$, were located distally on the long arm of pair 7 (7q). DAPI+ heterochromatin was predominantly centromeric, with some small pericentromeric bands. Although the C-banding patterns of other Bolitoglossa species are so far unknown, cytogenetic studies conducted in other Plethodontid salamanders have demonstrated that pericentromeric heterochromatin is a useful cytological marker for identifying interspecific homeologies. Species diversification is usually accompanied by chromosomal changes. Therefore, the cytogenetic characterization of Bolitoglossa populations from the middle and western Brazilian Amazon Basin could identify differences which may lead to the identification of new species.
\end{abstract}

Keywords: Salamanders, Bolitoglossa, karyotypes, Brazilian Amazon, cytogenetics.

Received: January 10, 2014; Accepted: June 4, 2014.

The Plethodontid salamander genus Bolitoglossa is, by far, the most species-rich and diverse genus of salamanders. Its 124 assigned species represent around $20 \%$ of the living caudate species (Sessions, 2008; Rovito et al., 2012; Frost, 2013; Brcko et al., 2013). Widespread over the American tropics, these salamanders are found from northeastern Mexico to the South American Rainforest, and Middle America is the most species-rich region (Parra-Olea et al., 2004). Cytogenetic information on Bolitoglossa species is scarce, being limited to 14 species (Green and Sessions, 2007). Like several other genera of the Plethodontidae family, the Bolitoglossa species studied show karyotypes with $2 \mathrm{n}=26$ bi-armed chromosomes and slight cytogenetic differences, basically in the location of Nucleolar Organizer Regions (NORs), with no available C-banding data (Sessions and Kezer, 1991). Based on a phylogenetic analysis of molecular data, Parra-Olea et al. (2004)

Send correspondence to Julio Cesar Pieczarka. Instituto de Ciências Biológicas, Universidade Federal do Pará, Campus Guamá, Avenida Perimetral s.n., Guamá, 66075-900 Belém, PA, Brazil. E-mail: juliopieczarka@gmail.com, julio@ufpa.br. removed the Brazilian salamander populations from the synonymy of $B$. altamazonica, resurrecting $B$. paraensis, which remain as the only formally recognized species in the Brazilian Amazon Rainforest. Furthermore, based on the large genetic divergences found among the Brazilian salamander samples studied by them, these authors also suggest the existence of a species complex under this taxon name, stating the need of more detailed studies. Recently, Brcko et al. (2013) analyzed the external morphological characteristics of 278 Bolitoglossa specimens and confirmed the presence of a species complex in the Brazilian Amazon, suggesting an increase of species from one to five ( $B$. caldwellae sp. nov., B. madeira sp. nov., B. tapajonica sp. nov., B. paraensis and B. altamazonica). In an attempt to find cytological markers capable of identifying interspecific differences, we present here a cytogenetic analysis of $B$. paraensis specimens from a type locality, aiming to find characteristics which might complement the cytogenetic data available for the genus and contribute to its cytotaxonomy. 
Twelve individuals belonging to the species Bolitoglossa paraensis were cytogenetically analyzed, ten of which were males (MPEG 21316-7, 21370, 22177-8, 31654-7, 31665; PS-518) and one female (MPEG 31656). Specimens collected in the Gunma Ecologic Park (municipality of Santa Barbara, State of Pará, Brazil; $1^{\circ} 12^{\prime} 46^{\prime \prime} \mathrm{S} / 48^{\circ} 17^{\prime} 18^{\prime \prime} \mathrm{W}$ ) were sacrificed with $5 \%$ lidocaine, fixed in $10 \%$ formalin, stored in $70 \%$ ethanol, and deposited in the herpetological collection of the "Museu Paraense Emilio Goeldi" (MPEG) and in the herpetological collection of the Cytogenetics Laboratory at the Federal University of Pará in Belém/PA. Specimens were collected under permanent permit number IBAMA 13248-1.

Preparations of mitotic and meiotic chromosomes were obtained, respectively, from intestine fragments and testes, according to Kezer et al. (1980). Chromosome preparations were placed onto slides and stained with a $5 \%$ Giemsa solution with phosphate buffer. Metaphase chromosomes were treated for C-banding (Sumner, 1972), submitted to $\mathrm{AgNO}_{3}$ impregnation (Howell and Black, 1980), staining with $\mathrm{CMA}_{3} / \mathrm{DAPI}$ fluorochromes (Schweizer and Ambros, 1994), and fluorescent in situ hybridization (FISH) (Viegas-Péquignot, 1992). Staining with $\mathrm{CMA}_{3} / \mathrm{DAPI}$ fluorochromes was done on cells previously submitted to C-banding or FISH procedures. Telomeric FISH experiments were performed with a DAKO FITC telomere PNA FISH kit (K532511-8; DAKO, Glostrup, Denmark), following the manufacturer's protocol. The karyotypes were organized using Adobe Photoshop CS5, and the chromosomes classified according to Green and Sessions (2007). Chromosome measurements and centromeric index $(\mathrm{CI})$ calculation of each chromosome pair were performed in 10 metaphases, using the Micromeasure v. 3.3 software (Reeves and Tear, 2000).

All B. paraensis specimens analyzed showed a karyotype with 13 chromosome pairs $(2 n=26, \mathrm{NF}=52)$, most of them metacentric, with the exception of pairs 9 and 12, which were submetacentric (Figure 1A, Table 1). Their C-banding pattern is characterized by a large amount of heterochromatin in all centromeric regions and small pericentromeric bands on both arms of pairs 1-9 (Figure 1B). There were no differences in C-banding patterns between males and females, indicating that there are no differentiated sex chromosomes in this species. Fluorochrome heterochromatin characterization with $\mathrm{CMA}_{3} / \mathrm{DAPI}$ techniques showed only DAPI+ bands (Figure 1C). Nucleolar Organizer Regions can also be evidenced by $\mathrm{CMA}_{3}$ (Green and Sessions, 2007), and in B. paraensis they were located distally on 7q, where the $\mathrm{CMA}_{3}+$ regions (Figure 1D) are coincident with the Ag-NORs labeling (Figure 2A-C). The meiotic cells analyzed showed 13 bivalents in diakinesis, with the same DAPI/CMA 3 banding pattern as the mitotic chromosomes (Figure 2B,C). FISH staining with telomere PNA probes showed a signal in the terminal regions of all

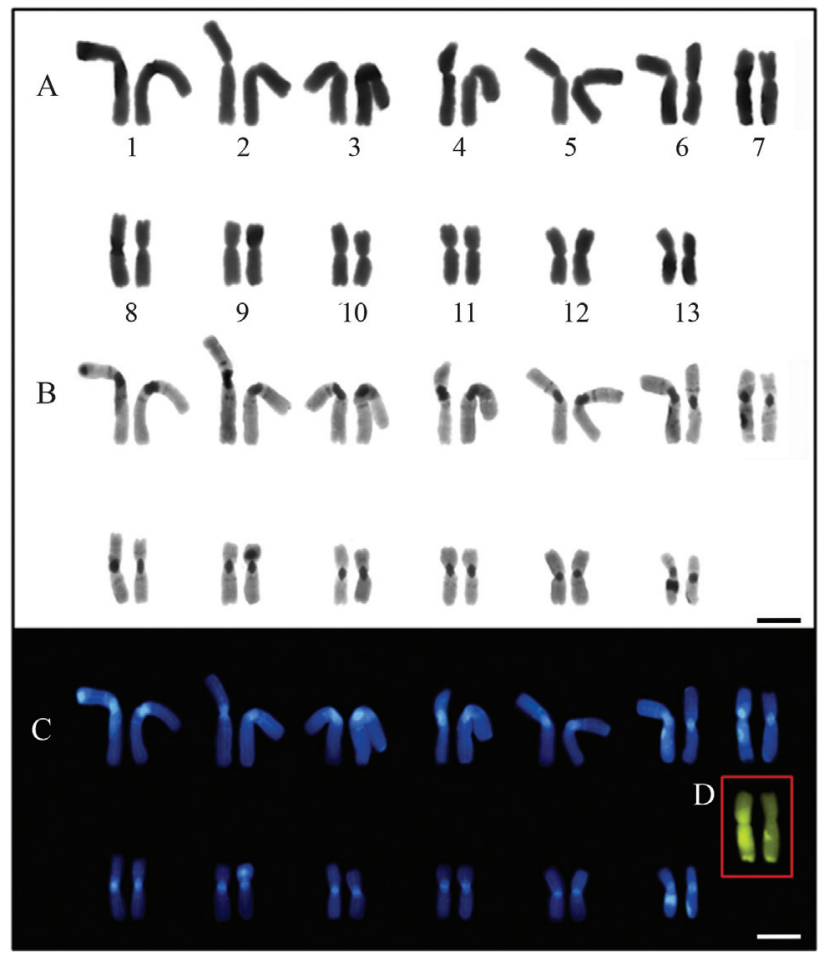

Figure 1 - Sequential karyotypes of a male specimen of Bolitoglossa paraensis: A) conventional Giemsa staining, B) C-banding pattern, C) DAPI staining; D) NOR $\mathrm{CMA}_{3}+$ staining. Bar $=10 \mu \mathrm{m}$.

Table 1 - Morphometric data of Bolitoglossa paraensis chromosomes. $(\mathrm{CI}=$ centromeric index; $\%$ set $=$ relative size; chromosome type: $\mathrm{m}=$ metacentric, $\mathrm{sm}=$ submetacentric; $*=$ NOR-bearing chromosome pair).

\begin{tabular}{lccc}
\hline Chromosome pair & \% set & CI & Type \\
\hline 1 & 11.13 & $0.434 \pm 0.025$ & $\mathrm{~m}$ \\
2 & 10.63 & $0.442 \pm 0.021$ & $\mathrm{~m}$ \\
3 & 10.23 & $0.451 \pm 0.029$ & $\mathrm{M}$ \\
4 & 9.27 & $0.46 \pm 0.038$ & $\mathrm{~m}$ \\
5 & 8.71 & $0.462 \pm 0.025$ & $\mathrm{~m}$ \\
6 & 8.27 & $0.445 \pm 0.037$ & $\mathrm{~m}$ \\
7 & 7.1 & $0.375 \pm 0.01$ & $\mathrm{~m} *$ \\
8 & 6.56 & $0.455 \pm 0.015$ & $\mathrm{~m}$ \\
9 & 5.94 & $0.373 \pm 0.01$ & $\mathrm{sm}$ \\
10 & 5.77 & $0.467 \pm 0.015$ & $\mathrm{~m}$ \\
11 & 5.77 & $0.396 \pm 0.025$ & $\mathrm{~m}$ \\
12 & 5.48 & $0.362 \pm 0.015$ & $\mathrm{sm}$ \\
13 & 5.13 & $0.445 \pm 0.02$ & $\mathrm{~m}$ \\
\hline
\end{tabular}

chromosomes (Figure 3). Again, no heteromorphic sex chromosomes were identified.

The cytogenetic findings described here for $B$. paraensis are consistent with the $2 \mathrm{n}$ and FN previously reported for other Bolitoglossa species (Green and Sessions, 2007), indicating great karyotype uniformity among species of this genus. 


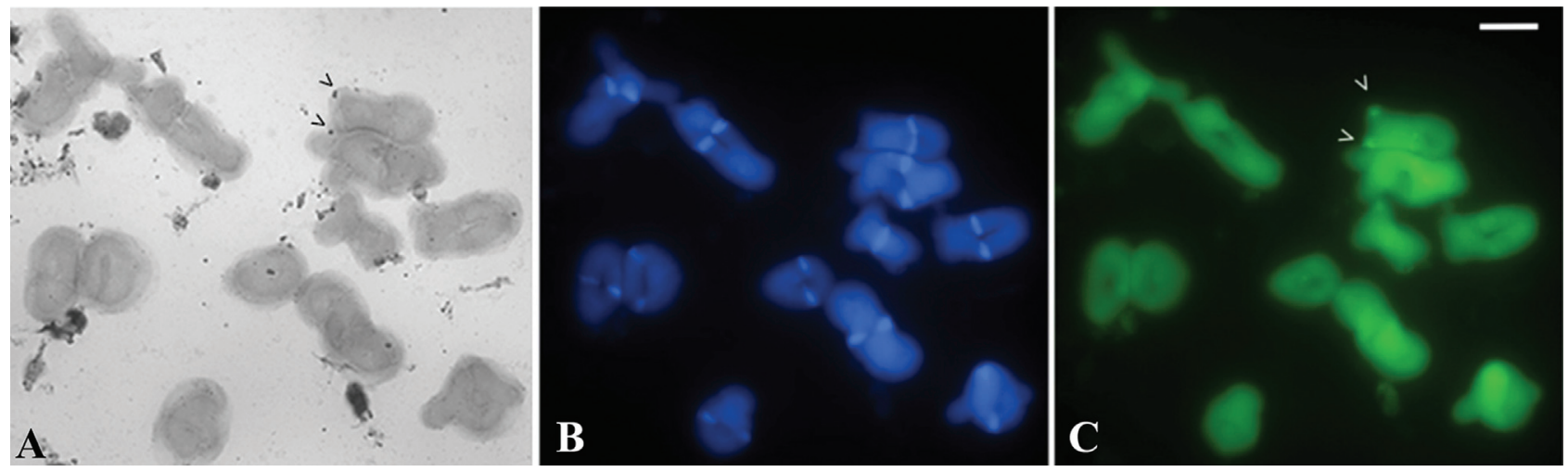

Figure 2 - Diakinesis: A) Ag-NOR, B) DAPI and C) $\mathrm{CMA}_{3}$ staining patterns of Bolitoglossa paraensis. Arrowheads indicate the locations of NORs. $\mathrm{Bar}=10 \mu \mathrm{m}$

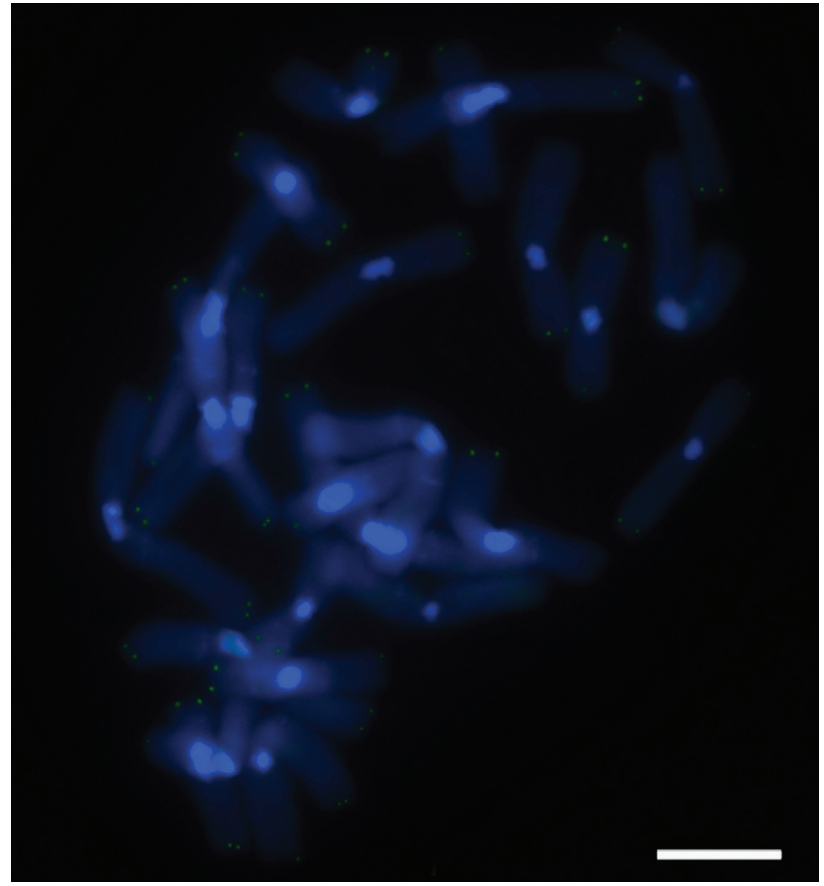

Figure 3 - FISH signals obtained with telomere PNA probes in the terminal regions of all chromosomes. Bar $=10 \mu \mathrm{m}$.

Sessions and Kezer (1991) analyzed the location of NORs in 26 species of Plethodontid salamanders, eight of them belonging to genus Bolitoglossa. These authors found 13 different NOR locations, which they classified from A to $\mathrm{M}$, also discriminating primary (present on both homologous chromosomes) and secondary (present on only one homolog) NORs. In Bolitoglossa species, primary NORs were found at locations A ( $B$. adspersa), B (B. engelhardti and B. helmrichi), and $\mathrm{H}$ (B. flaviventris, B. subpalmata, $B$. dofleini, B. franklini, and B. rostrata). The labeling location, relative size, and chromosome morphology of the NOR-bearing pair of $B$. paraensis resemble type $\mathrm{H}$ in the classification of Sessions and Kezer (1991). However, it is difficult to make comparisons, because the classification used by those authors did not associate NOR sites to any chromosome pair.

Although the C-banding patterns of other Bolitoglossa species are unknown, cytogenetic studies performed in other Plethodontid salamanders have demonstrated that pericentromeric heterochromatin is a useful cytological marker for identifying interspecific homeologies (Sessions, 2008). Several hypotheses have been proposed to explain the origin of pericentromeric heterochromatin in Caudata, and their relationship with satellite DNA (stDNA) appears to be well established. According to Macgregor and Sessions (1986), the growth and dispersion of stDNA located at centromeres are involved in the formation of pericentromeric bands. Under this model, stDNA would be accumulated in the centromeres by tandem duplication mechanisms as a first step, and chromosomal rearrangements, such as inversions and/or unequal exchanges at these sites, would cause random breaks and dispersion of stDNA over the chromosome arms, thus producing the pericentromeric bands. Hence, the age of stDNA is related to its chromosomal location, because stDNA present only in closely related species, considered "new", is found in centromeric regions, whereas "older" stDNA, shared by distant lineages, is located in interstitial or pericentromeric regions (for review, see Sessions 2008 and references therein). The available stDNA sequencing data show them to be AT-rich regions (Barsacchi-Pilone et al., 1986), so it is not surprising that the heterochromatin present in $B$. paraensis was DAPI+. Detailed studies of stDNA, such as its isolation and sequencing from pericentromeric and centromeric heterochromatic regions in B. paraensis by cloning and fluorescent in situ hybridization, can provide useful information about the karyotype evolution in this genus.

Telomeres are considered a conserved sequence (TTAGGG) among invertebrate and vertebrate species (Meyne et al., 1989). However, the absence of FISH hybridization signals using telomere probes in the newt Cynops pyrrhogaster led Murakami et al. (2007) to suggest that there could be differences in the telomeric repetitive 
consensus sequences of Caudata species, as observed in Drosophila species and plants of family Alliaceae (Fuchs et al., 1995; Biessmann et al., 2000). The results shown here for B. paraensis (Figure 3), however, do not support this assumption, or at least indicate that it is not extensive to all Caudata species.

The diversity of primary and secondary NOR sites, together with the qualitative and quantitative variability of stDNA, lead us to believe that a detailed cytogenetic and molecular characterization of Bolitoglossa species from the middle and western Brazilian Amazon Basin should disclose useful differences, not only improving our knowledge on chromosome evolution in Bolitoglossa species, but also helping to identify new species of this genus.

\section{Acknowledgments}

The authors acknowledge the financial support provided by Conselho Nacional de Desenvolvimento Científico e Tecnológico (CNPq), Museu Paraense Emilio Goeldi (MPEG) and Universidade Federal do Pará (UFPa). JCP and CYN were recipients of Research Scholarships 307071/2009-0 and 306989/2009-3, respectively, granted by $\mathrm{CNPq}$. PS was the recipient of a $\mathrm{PhD}$ scholarship (141771/2006-2) granted by CNPq. JBS had an undergraduate science training scholarship from CNPq. The authors are grateful for the field support provided by S. Neckel, J.F Sarmento, I.C Brcko and U. Galatti.

\section{References}

Barsacchi-Pilone G, Batisoni R, Andronico F, Vitelli L and Nardi I (1986) Heterochromatic DNA in Triturus (Amphibia, Urodela). I. A satellite DNA component of the pericentric Cbands. Chromosoma 93:435-446.

Biessmann H, Zurovcova M, Yao JG, Lozovskaya E and Walter MF (2000) A telomeric satellite in Drosophila virilis and its sibling species. Chromosoma 109:372-380.

Brcko IC, Hoogmoed MS and Neckel-Oliveira S (2013) Taxonomy and distribution of the salamander genus Bolitoglossa Duméril, Bibron \& Duméril, 1854 (Amphibia, Caudata, Plethodontidae) in Brazilian Amazonia. Zootaxa 3686:401431.

Fuchs J, Brandes A and Schubert I (1995) Telomere sequence localization and karyotype evolution in higher plants. Plant Syst Evol 196:227-241.

Green DM and Sessions SK (2007) Karyology and cytogenetics. In: Heatwole H (ed) Amphibian Biology. Volume 7. Surrey Beatty \& Sons, Chipping Norton, pp 2757-2842.
Howell WN and Black DA (1980) Controlled silver staining of nucleolus organizer regions with a protective colloidal developer: A one step method. Experientia 36:1014-1015.

Kezer J, Leon PE and Sessions SK (1980) Structural differentiation of the meiotic and mitotic chromosomes of the salamander, Ambystoma macrodactylum. Chromosoma 81:177-197.

Macgregor HC and sessions SK (1986). The biological significance of variation in satellite DNA and heterochromatin in newts of the genus Triturus: An evolutionary perspective. Phil Trans R Soc Lond B 312 86:243-259.

Meyne J, Ratliff RL and Moyzis RK (1989) Conservation of the human telomere sequence (TTAGGG)n among vertebrates. Proc Natl Acad Sci USA 86:7049-7053.

Murakami T, Maki N, Nishida-Umehara C, Matsuda Y and Agata K (2007) Establishment of high-resolution FISH mapping system and its application for molecular cytogenetic characterization of chromosomes in the newt Cynops pyrrhogaster (Urodela, Amphibia). Chromosome Res 15:471-484.

Parra-Olea G, García-París M and Wake DB (2004) Molecular diversification of salamanders of the tropical American genus Bolitoglossa (Caudata, Plethodontidae) and its evolutionary and biogeographical implications. Biol J Linn Soc Lond 81:325-346.

Rovito SM, Parra-Olea G, Lee D and Wake D (2012) A new species of Bolitoglossa (Amphibia, Caudata) from the Sierra de Juárez, Oaxaca, Mexico. Zoo Keys 185:55-71.

Schweizer D and Ambros PF (1994) Chromosome banding. In: Gosden JR (ed). Methods in Molecular Biology. Humana Press, Totowa, pp 97-112.

Sessions SK (2008) Evolutionary cytogenetics in salamanders. Chromosome Res 16:183-201.

Sessions SK and Kezer J (1991) Evolutionary cytogenetics of bolitoglossine salamanders (Family Plethodontiade). In: Green DM and Sessions SK (eds) Amphibian Cytogenetics and Evolution. Academic Press, San Diego, pp 89-130.

Sumner AT (1972) A simple technique for demostrating centromeric heterochromatin. Exp Experimental Cell Research 75 72:304-306.

Viegas-Pequignot E (1992) In situ hybridization to chromosomes with biotinylated probes. In: Wilkinson DG (ed) In situ Hybridization: A Practical Approach. IRL Press, Oxford, pp 137-158.

\section{Internet Resources}

Frost DR (2013) Amphibian Species of the World: an Online Reference. Version 5.6, American Museum of Natural History, New York, USA, http://research.amnh.org/herpetology/amphibia/index.html (9 January, 2013).

Reeves A and Tear J (2000) Micro Measure for Windows, version 3.3, http://www.colostate.edu/Depts/Biology/MicroMeasure (14 August, 2012).

Associate Editor: Yatiyo Yonenaga-Yassuda

License information: This is an open-access article distributed under the terms of the Creative Commons Attribution License, which permits unrestricted use, distribution, and reproduction in any medium, provided the original work is properly cited. 